\title{
Asymptotic estimates and nonexistence results for critical problems with Hardy term involving Grushin-type operators
}

\author{
Annunziata Loiudice ${ }^{1}$
}

Received: 23 October 2018 / Accepted: 23 March 2019 / Published online: 2 April 2019

(c) Fondazione Annali di Matematica Pura ed Applicata and Springer-Verlag GmbH Germany, part of Springer Nature 2019

\begin{abstract}
We provide the asymptotic behavior of solutions, at the singularity and at infinity, for a class of subelliptic Dirichlet problems with Hardy perturbation and critical nonlinearity of the type $-\mathcal{L}_{\alpha} u-\mu \frac{\psi^{2}}{d^{2}} u=K(z)|u|^{2^{*}-2} u$ in $\Omega$, where $\mathcal{L}_{\alpha}=\Delta_{x}+|x|^{2 \alpha} \Delta_{y}, \alpha>0$ is the so-called Grushin operator, $\Omega$ is an open subset of $\mathbb{R}^{N}, 0 \in \Omega, d$ is the gauge norm naturally associated with $\mathcal{L}_{\alpha}, \psi:=\left|\nabla_{\alpha} d\right|$, where $\nabla_{\alpha}$ is the Grushin gradient, $K \in L^{\infty}$ and $0 \leq \mu<\bar{\mu}$, where $\bar{\mu}$ is the best Hardy constant for $\mathcal{L}_{\alpha}$. Furthermore, we establish some Pohozaev-type nonexistence results.
\end{abstract}

Keywords Subelliptic critical problem · Grushin operator · Hardy potential · Asymptotic behavior · Pohozaev-type identity $\cdot$ Nonexistence results

Mathematics Subject Classification $35 \mathrm{~J} 70 \cdot 35 \mathrm{~J} 75 \cdot 35 \mathrm{~B} 40$

\section{Introduction}

The purpose of this paper is to investigate regularity, asymptotic behavior and nonexistence results for a class of semilinear equations with Hardy term involving the so-called Grushin operator, defined on $\mathbb{R}^{N}=\mathbb{R}_{x}^{m} \times \mathbb{R}_{y}^{n}$ by

$$
\mathcal{L}_{\alpha}=\Delta_{x}+|x|^{2 \alpha} \Delta_{y}, \quad \alpha>0 .
$$

We recall that this operator is elliptic for $x \neq 0$ and degenerates on the manifold $\{0\} \times \mathbb{R}^{n}$. When $\alpha$ is a nonnegative integer, this operator falls into the class of Hörmander-type operators; in the general case, it belongs to the wide class of subelliptic operators studied by Franchi and Lanconelli in [11-13]. Moreover, it constitutes the simplest prototype of the so-called $\Delta_{\lambda}$-operators introduced by Kogoj and Lanconelli in [20] and it also belongs to the more general class of $X$-elliptic operators studied in [19].

Annunziata Loiudice

annunziata.loiudice@uniba.it

1 Dipartimento di Matematica, Università degli Studi di Bari, Via Orabona, 4, 70125 Bari, Italy 
Let

$$
X_{i}=\frac{\partial}{\partial x_{i}}, \quad i=1, \ldots, m, \quad X_{m+j}=|x|^{\alpha} \frac{\partial}{\partial y_{j}}, \quad j=1, \ldots, n
$$

and

$$
\nabla_{\alpha}=\left(X_{1}, \ldots, X_{N}\right) .
$$

For a given open subset $\Omega \subset \mathbb{R}^{N}$, we shall indicate by $\mathcal{D}_{\alpha}^{1,2}(\Omega)$ the completion of $C_{0}^{\infty}(\Omega)$ with respect to the norm

$$
\|u\|:=\int_{\Omega}\left|\nabla_{\alpha} u\right|^{2} \mathrm{~d} z
$$

Let, moreover,

$$
Q=m+(\alpha+1) n
$$

denote the homogeneous dimension naturally attached to the operator $\mathcal{L}_{\alpha}$ (see Sect. 2).

Our aim is to study qualitative properties of solutions in $\mathcal{D}_{\alpha}^{1,2}(\Omega)$ to the following class of semilinear subelliptic equations with Hardy term and critical nonlinearity

$$
\begin{cases}-\mathcal{L}_{\alpha} u-\mu \frac{\psi^{2}}{d^{2}} u=K(z)|u|^{2^{*}-2} u & \text { in } \Omega, \\ u=0 & \text { on } \partial \Omega\end{cases}
$$

where $\Omega \subset \mathbb{R}^{N}=\mathbb{R}^{m} \times \mathbb{R}^{n}$ is an arbitrary open set, $0 \in \Omega, d$ is the natural gauge associated with $\mathcal{L}_{\alpha}$, defined, for $z=(x, y) \in \mathbb{R}^{m} \times \mathbb{R}^{n}$, by

$$
d(z)=\left(|x|^{2(\alpha+1)}+(\alpha+1)^{2}|y|^{2}\right)^{\frac{1}{2(\alpha+1)}},
$$

$\psi:=\left|\nabla_{\alpha} d\right|, K$ is a bounded coefficient, $2^{*}=\frac{2 Q}{Q-2}$ denotes the critical Sobolev exponent in this context and $0 \leq \mu<\bar{\mu}$, where $\bar{\mu}=\left(\frac{Q-2}{2}\right)^{2}$ is the best constant in the Hardy inequality for the Grushin gradient

$$
\int_{\Omega}\left|\nabla_{\alpha} u\right|^{2} \mathrm{~d} z \geq \bar{\mu} \int_{\Omega} \psi^{2} \frac{|u|^{2}}{d(z)^{2}} \mathrm{~d} z, \quad \forall u \in C_{0}^{\infty}(\Omega) .
$$

Precisely, we establish the exact asymptotic behavior at the singularity and at infinity, in the case of unbounded domains, of finite energy solutions to problem (1.2), extending the asymptotic results obtained for the case $\mu=0$ by the author in [25]. Moreover, by means of Pohozaev-type arguments, we provide nonexistence results for problem (1.2) on bounded starshaped domains with respect to the Grushin geometry and necessary conditions for the existence of entire solutions on the whole $\mathbb{R}^{N}$.

We recall that the Hardy-type inequality (1.3) involving the Grushin gradient, which is the main functional tool in the variational formulation of problem (1.2), was proved by Garofalo in [14]; we also quote [4,21] for related results and generalizations. In particular, in [4], among other results, it is proved that, when $0 \in \Omega$, the constant $\bar{\mu}=(Q-2)^{2} / 4$ is the best constant in (1.3) and it is never attained; in [21], the validity of the above inequality is extended to the general class of the so-called $\Delta_{\lambda}$-operators. Some Hardy inequalities with remainder terms can also be found in [41].

Note that the Dirichlet problem involving the operator $\mathcal{L}_{\alpha}$ has been deeply studied by many authors under different perspectives (see, e.g., [2,5,14,20,22,23,30,33,34,38-40] and the references therein). In particular, Monti and Morbidelli in [33] investigate uniqueness and symmetry of solutions for the unperturbed critical problem involving the Grushin operator 
and introduce a suitable Kelvin-type transform in this context, which will be a useful tool in our asymptotic analysis; in [20], Kogoj and Lanconelli provide regularity, existence and Pohozaev-type nonexistence results for the Dirichlet problem $-\mathcal{L}_{\alpha} u=f(u)$ for general nonlinearities $f$, in the larger framework of $\Delta_{\lambda}$-operators.

In this paper, we focus on the critical problem (1.2). The first part of the paper is devoted to study the singularity of solutions at 0 . The result is established for a general class of nonlinearities possessing at most critical growth. In what follows, $B_{r}$ will indicate the ball with center at 0 and radius $r$ with respect to the homogeneous norm $d$.

Theorem 1.1 Let $\Omega \subset \mathbb{R}^{N}$ be an arbitrary open set, $0 \in \Omega$. If $u \in \mathcal{D}_{\alpha}^{1,2}(\Omega)$ is a weak solution to problem

$$
-\mathcal{L}_{\alpha} u-\mu \frac{\psi^{2}}{d^{2}} u=f(z, u) \text { in } \Omega
$$

where $f: \Omega \times \mathbb{R} \rightarrow \mathbb{R}$ is a Carathéodory function such that

$$
|f(z, t)| \leq C\left(|t|+|t|^{2^{*}-1}\right), \quad \forall t \in \mathbb{R},
$$

there exist positive constants $C$ and $r$ such that

$$
|u(z)| \leq \frac{C}{d(z)^{\sqrt{\bar{\mu}}-\sqrt{\bar{\mu}-\mu}}}, \quad \forall z \in B_{r} \backslash\{0\} .
$$

If, moreover, $u$ is positive and $f(z, t) \geq 0$ for $t>0$, there exist positive constants $C^{\prime}$ and $r^{\prime}$ such that

$$
u(z) \geq \frac{C^{\prime}}{d(z)^{\sqrt{\mu}-\sqrt{\mu-\mu}}}, \quad \forall z \in B_{r^{\prime}} \backslash\{0\} .
$$

The above asymptotic estimates in the Euclidean canonical setting go back to $[3,8,17]$, and they have been an interesting tool in the study of many related problems. Recently, this kind of estimates has been extended by the author in [28] to the subelliptic framework of sub-Laplacians on Carnot groups. Following the Euclidean scheme, the estimate from above is achieved by means of Moser-type estimates and the estimate from below relies on a suitable comparison argument. We observe that the above general theorem also provides an estimate of the singularity of the $L^{2}$-normalized eigenfunctions of the operator $-\mathcal{L}_{\alpha}-\mu \frac{\psi^{2}}{d^{2}}$ with Dirichlet boundary conditions, whose existence can be easily proved as in the Heisenberg case following [31].

Now, when $f$ is a purely critical nonlinearity and $\Omega$ is a neighborhood of $\infty$, by exploiting the conformal invariance of the equation, from the behavior at 0 , we can deduce the exact asymptotic decay of solutions at infinity by means of the Kelvin-type transform introduced in [33]. The complete description of the asymptotics in this case is contained in the following theorem.

Theorem 1.2 Let $\Omega \subset \mathbb{R}^{N}$ be a neighborhood of $\infty, 0 \in \Omega$ and let $K \in L^{\infty}$ be nonnegative. If $u \in \mathcal{D}_{\alpha}^{1,2}(\Omega)$ is a positive solution to

$$
-\mathcal{L}_{\alpha} u-\mu \frac{\psi^{2}}{d^{2}} u=K(z) u^{2^{*}-1} \text { in } \Omega,
$$

then $u$ satisfies

$$
\begin{aligned}
& u(z) \sim \frac{1}{d(z)^{\sqrt{\bar{\mu}}-\sqrt{\bar{\mu}-\mu}}, \quad \text { as } \quad d(z) \rightarrow 0,} \\
& u(z) \sim \frac{1}{d(z)^{\sqrt{\bar{\mu}}+\sqrt{\bar{\mu}-\mu}}, \quad \text { as }} d(z) \rightarrow \infty .
\end{aligned}
$$


We remark that in the ordinary Laplacian case, when $K \equiv 1$ and $\Omega=\mathbb{R}^{N}$, all positive solutions to (1.8) are known to have the explicit form

$$
U_{\epsilon}(x)=\frac{[4 \epsilon(\bar{\mu}-\mu) N(N-2)]^{\frac{N-2}{4}}}{\left[\epsilon|x|^{\gamma^{\prime} / \sqrt{\mu}}+|x|^{\gamma / \sqrt{\mu}}\right]^{\frac{N-2}{2}}}, \quad \epsilon>0,
$$

where $\gamma^{\prime}=\sqrt{\bar{\mu}}-\sqrt{\bar{\mu}-\mu}$ and $\gamma=\sqrt{\bar{\mu}}+\sqrt{\bar{\mu}-\mu}$ (see Terracini [37], Jannelli [18]). So, our estimates in the Grushin case reflect the behavior of the functions $U_{\epsilon}$ in the Euclidean framework. We recall that the above result in the case $\mu=0$ was obtained by the author in [25], where it was proved that the finite energy solutions of (1.8) in the unperturbed case are bounded at 0 and decay at infinity like $d^{2-Q}$, which is the fundamental solution with pole at 0 of the involved operator (see also [26,28,29] and the references therein for analogous decay results concerning sub-Laplacians on Carnot groups).

The second part of the paper is devoted to some nonexistence results for positive solutions to problem (1.2), which are obtained by means of Pohozaev-type arguments. A first nonexistence result concerns bounded starshaped domains with respect to the Grushin geometry, and it extends to the present singular case analogous results proved by Kogoj and Lanconelli in [20] for the case $\mu=0$ and autonomous nonlinearities in the larger framework of $\Delta_{\lambda}$ operators. We first recall the definition of $\delta_{\lambda}$-starshaped domains, where $\delta_{\lambda}$ denotes the family of anisotropic dilations naturally attached to the homogeneity of the operator $\mathcal{L}_{\alpha}$, i.e.,

$$
\delta_{\lambda}(x, y)=\left(\lambda x, \lambda^{\alpha+1} y\right), \quad \lambda>0 .
$$

In what follows, $Z$ will indicate the infinitesimal generator of the dilations $\delta_{\lambda}$, whose expression is recalled in formula (2.4) below.

Definition 1.3 Let $\Omega \subset \mathbb{R}^{N}$ be a connected open set with $C^{1}$-boundary, containing 0 at its interior. We say that $\Omega$ is $\delta_{\lambda}$-starshaped with respect to the origin if

$$
\langle Z, v\rangle(z) \geq 0 \forall z \in \partial \Omega,
$$

where $v$ denotes the outer unit normal to the boundary of $\Omega$.

Our nonexistence result on bounded starshaped domains is the following (see Sect. 2 for the definition of the functional space $\Lambda^{2}$ appearing in the statement of the theorem):

Theorem 1.4 Let $\Omega \subset \mathbb{R}^{N}$ be a connected bounded domain with $C^{1}$-boundary, $\delta_{\lambda}$ starshaped about the origin. Assume that $K \in C^{1}(\bar{\Omega})$ and $Z K \leq 0$ in $\Omega$. Then, the problem

$$
-\mathcal{L}_{\alpha} u-\mu \frac{\psi^{2}}{d^{2}} u=K(z) u^{2^{*}-1} \text { in } \Omega, \quad u=0 \text { on } \partial \Omega
$$

has no nonnegative nontrivial solutions $u \in \mathcal{D}_{\alpha}^{1,2}(\Omega) \cap \Lambda^{2}(\bar{\Omega} \backslash\{0\})$.

The result applies, in particular, if $K$ is constant or, more generally, homogeneous of degree zero with respect to the dilations $\delta_{\lambda}$, in which case $Z K \equiv 0$ in $\Omega$.

Finally, concerning entire solutions of the problem in the whole $\mathbb{R}^{N}$, we prove the following result.

Theorem 1.5 Assume that $K \in L^{\infty}\left(\mathbb{R}^{N}\right) \cap C^{1}\left(\mathbb{R}^{N}\right), Z K \in L^{\infty}\left(\mathbb{R}^{N}\right)$ and let $u \in \mathcal{D}_{\alpha}^{1,2}\left(\mathbb{R}^{N}\right)$ be a positive solution to

$$
-\mathcal{L}_{\alpha} u-\mu \frac{\psi^{2}}{d^{2}} u=K(z) u^{2^{*}-1} \text { in } \mathbb{R}^{N} .
$$


Then,

$$
\int_{\mathbb{R}^{N}} Z K \cdot u^{2^{*}} \mathrm{~d} z=0
$$

The above condition implies that there are no positive solutions if the radial derivative $Z K$ of the coefficient $K$ does not change sign in $\mathbb{R}^{N}$, and $K$ is not $\delta_{\lambda}$ homogeneous of degree 0 . We remark that the necessary condition (1.14) for the existence of positive solutions to the critical problem (1.13) is the Grushin counterpart of the Euclidean condition

$$
\int_{\mathbb{R}^{N}}\langle\nabla K(x), x\rangle u^{2^{*}} \mathrm{~d} x=0
$$

which appears in Smets [36, Proposition 2.2] and Felli-Schneider [10, Corollary 2.3].

The paper is organized as follows. In Sect. 2, we introduce the functional setting of Grushin-type operators and their main properties; in Sect. 3, we study the behavior at the singularity of solutions to our problem, as stated in Theorem 1.1; in Sect. 4, we focus on the critical equation and we derive the asymptotic behavior of solutions at infinity on exterior domains on $\mathbb{R}^{N}$ from the behavior at 0 by means of the Kelvin transform for the Grushin gradient; finally, in Sect. 5, we provide some weighted Pohozaev-type identities related to the involved operator and, consequently, we derive the nonexistence results stated in Theorems 1.4 and 1.5.

\section{Preliminaries and notation}

Let us begin by recalling that the operator $\mathcal{L}_{\alpha}=\Delta_{x}+|x|^{2 \alpha} \Delta_{y}$ is a degenerate elliptic operator, which is homogeneous of degree two with respect to the family of anisotropic dilations $\delta_{\lambda}$ defined in (1.11). The number

$$
Q=m+(\alpha+1) n,
$$

naturally attached to the dilations $\delta_{\lambda}$, plays the rôle of a global space dimension in the analysis of the operator $\mathcal{L}_{\alpha}$, and it is usually called the homogeneous dimension of the space $\mathbb{R}^{N}=\mathbb{R}^{m} \times \mathbb{R}^{n}$ with respect to $\delta_{\lambda}$.

Denoted by $\nabla_{\alpha}=\left(X_{1}, \ldots, X_{N}\right)$ the Grushin gradient, where the $X_{j}$ 's are defined in (1.1), for any $h=\left(h_{1}, \ldots, h_{N}\right) \in C^{1}\left(\mathbb{R}^{N}, \mathbb{R}^{N}\right)$, we shall indicate by

$$
\operatorname{div}_{\alpha}(h)=\sum_{i=1}^{N} X_{i} h_{i}
$$

the divergence induced by the Grushin vector fields. With this notation, $\mathcal{L}_{\alpha}=\operatorname{div}_{\alpha} \nabla_{\alpha}$. In what follows, it will be convenient to consider $\mathcal{L}_{\alpha}$ as a divergence form operator on $\mathbb{R}^{N}$. To this aim, consider the $N \times N$ matrix

$$
A_{\alpha}=\left(\begin{array}{c|c}
I_{\mathbb{R}^{m}} & 0 \\
\hline 0 & |x|^{2 \alpha} I_{\mathbb{R}^{n}}
\end{array}\right)
$$

Then,

$$
\mathcal{L}_{\alpha}=\operatorname{div}(\mathrm{A} \nabla)
$$


where div and $\nabla$ are the usual Euclidean operators taken with respect to the variable $z \in \mathbb{R}^{N}$. Observe that, for a function $u \in C^{1}\left(\mathbb{R}^{N}\right)$, it holds

$$
\left|\nabla_{\alpha} u\right|^{2}=\left\langle A_{\alpha} \nabla u, \nabla u\right\rangle=\left|\nabla_{x} u\right|^{2}+|x|^{2 \alpha}\left|\nabla_{y} u\right|^{2} .
$$

For $z=(x, y) \in \mathbb{R}^{m} \times \mathbb{R}^{n}$, let

$$
d(z)=d(x, y)=\left(|x|^{2(\alpha+1)}+(\alpha+1)^{2}|y|^{2}\right)^{\frac{1}{2(\alpha+1)}} .
$$

The function $d(z)$ is homogeneous of degree one with respect to the anisotropic dilations (1.11). Moreover, there exists a suitable constant $C>0$ depending on $\alpha$ and $Q$, such that

$$
\Gamma(z)=\frac{C}{d(z)^{Q-2}}
$$

is the fundamental solution of $-\mathcal{L}_{\alpha}$ with pole at the origin (see [14, Proposition 2.1]).

For $R>0$, we shall denote by $B_{d}(0, R)$, or simply $B_{R}$, the ball with center at 0 and radius $R$ with respect to the homogeneous norm $d$, i.e.,

$$
B_{R}=\left\{z \in \mathbb{R}^{N} \mid d(z)<R\right\} .
$$

We recall that, if we let $\psi:=\left|\nabla_{\alpha} d\right|$, then $\psi$ has the following explicit form

$$
\psi(z)=\frac{|x|^{\alpha}}{d(z)^{\alpha}} .
$$

Such weight function naturally appears in the Hardy-type inequality for $\mathcal{L}_{\alpha}$ recalled in (1.3). Moreover, if $f=f(d) \in C^{2}\left(\mathbb{R}^{+}\right)$is a radial function with respect to $d$, the following formula holds

$$
\mathcal{L}_{\alpha}(f(d))=\psi^{2}\left\{f^{\prime \prime}(d)+\frac{Q-1}{d} f^{\prime}(d)\right\} .
$$

(see, e.g., [14, formula (2.19)]).

We recall that if $u$ is a function on $\mathbb{R}^{N}$, we say that $u$ is homogeneous of degree $k \in \mathbb{R}$ with respect to the dilations (1.11) if for any $\lambda>0$

$$
u \circ \delta_{\lambda}=\lambda^{k} u \text {. }
$$

In Sect. 5, we shall consider the following smooth vector field on $\mathbb{R}^{N}$

$$
Z=\sum_{i=1}^{m} x_{i} \frac{\partial}{\partial x_{i}}+(\alpha+1) \sum_{j=1}^{n} y_{j} \frac{\partial}{\partial y_{j}}
$$

which is related to Grushin geometry since it is the infinitesimal generator of the dilations $\delta_{\lambda}$, so that $u$ satisfies (2.3) if and only if $Z u=k u$. In particular, since $d$ is homogeneous of degree one with respect to $\delta_{\lambda}$, then

$$
Z d=d
$$

Letting $2^{*}=\frac{2 Q}{Q-2}$, the following global Sobolev-type inequality holds

$$
\int_{\mathbb{R}^{N}}|u|^{2^{*}} \mathrm{~d} z \leq C \int_{\mathbb{R}^{N}}\left|\nabla_{\alpha} u\right|^{2} \mathrm{~d} z, \quad \forall u \in C_{0}^{\infty}\left(\mathbb{R}^{N}\right)
$$

where $C$ is a positive constant depending on $Q$. This inequality can be derived as a consequence of the general embedding results by Franchi and Lanconelli in [13], as explicitly verified by the author in [24], where also Sobolev inequalities with remainder terms for the 
Grushin gradient have been obtained. We quote [20] for the general Sobolev embedding inequality for the $\Delta_{\lambda}$-operators; we also refer to [1,32] for further related results.

In our treatment, we shall need some weighted versions of the above Sobolev inequality which we here recall. If $0 \leq s \leq 2$ and $a>\frac{2-Q}{2}$, there exists a positive constant $C$ depending on $s, a, Q$ such that

$$
\left(\int_{\mathbb{R}^{N}} \psi^{s} \frac{\left|d^{a} u\right|^{2^{*}(s)}}{d^{s}} \mathrm{~d} z\right)^{\frac{2}{2^{*}(s)}} \leq C \int_{\mathbb{R}^{N}}\left|d^{a} \nabla_{\alpha} u\right|^{2} \mathrm{~d} z, \quad \forall u \in C_{0}^{\infty}\left(\mathbb{R}^{N}\right)
$$

where $2^{*}(s)=\frac{2(Q-s)}{Q-2}$. The above inequality in the case $a=0$ is a Hardy-Sobolev-type inequality, and it can be easily derived by combining Hardy and Sobolev inequality for the Grushin gradient; a direct proof can be found in Dou and Niu [6]. The present weighted version can be easily obtained reasoning as in the Appendix of [28], where the analogous inequality for the horizontal gradient on stratified Lie groups is obtained.

We conclude by introducing the functional spaces, we shall use throughout the paper. If $\Omega$ is an open subset of $\mathbb{R}^{N}$, we shall indicate by $\Lambda^{2}(\bar{\Omega} \backslash\{0\})$ the space of functions $u \in \mathcal{C}(\bar{\Omega} \backslash\{0\})$ such that $X_{j} u, X_{j}^{2} u \in \mathcal{C}(\bar{\Omega} \backslash\{0\})$, for $j=1, \ldots, N$. The space $\mathcal{D}_{\alpha}^{1,2}\left(\Omega, d^{-2 \beta} \mathrm{d} z\right)$, or simply $\mathcal{D}_{\alpha, \beta}^{1,2}(\Omega)$, with $\beta<\frac{Q-2}{2}$, will denote the completion of $C_{0}^{\infty}(\Omega)$ with respect to the weighted norm

$$
\|u\|_{\mathcal{D}_{\alpha, \beta}^{1,2}(\Omega)}:=\int_{\Omega} d^{-2 \beta}\left|\nabla_{\alpha} u\right|^{2} \mathrm{~d} z .
$$

Moreover, we shall indicate by $S_{\alpha, \beta}^{1,2}(\Omega)$ the space of functions $u \in L^{2^{*}}\left(\Omega, d^{-2^{*} \beta} \mathrm{d} z\right)$ such that $\nabla_{\alpha} u \in L^{2}\left(\Omega, d^{-2 \beta} \mathrm{d} z\right)$ endowed with the norm

$$
\|u\|_{S_{\alpha, \beta}^{1,2}(\Omega)}:=\int_{\Omega} d^{-2^{*} \beta}|u|^{2^{*}} \mathrm{~d} z+\int_{\Omega} d^{-2 \beta}\left|\nabla_{\alpha} u\right|^{2} \mathrm{~d} z .
$$

In view of (2.6) for $s=0$ and $a=-\beta$, the two norms are equivalent on $C_{0}^{\infty}(\Omega)$.

\section{Asymptotic behavior at the origin}

In order to state the behavior of solutions to problem (1.4) at the origin, we first need to study their $L^{p}$ regularity. The following Brezis-Kato-type result holds.

Lemma 3.1 Let $\Omega \subset \mathbb{R}^{N}$ be a bounded neighborhood of 0 and $0 \leq \mu<\bar{\mu}$. Assume that $V \in L^{Q / 2}(\Omega)$ and $g \in L^{q}(\Omega), q \geq 2$. If $u \in \mathcal{D}_{\alpha}^{1,2}(\Omega)$ is a weak solution of

$$
-\mathcal{L}_{\alpha} u-\mu \frac{\psi^{2}}{d^{2}} u-V u+v u=g \text { in } \Omega,
$$

where $v$ is such that the linear operator on the left-hand side is positive, then

$$
u \in L^{p}(\Omega), \quad \forall p<p_{\lim }, \text { where } p_{\lim }=2^{*} \min \left\{\frac{q}{2}, \frac{\sqrt{\bar{\mu}}}{\sqrt{\bar{\mu}}-\sqrt{\bar{\mu}-\mu}}\right\} .
$$

Proof The proof follows the Euclidean outline in Smets [36], and it is based on the validity of Hardy and Sobolev inequality for the Grushin gradient (see also [28] for the analogous proof for sub-Laplacians on stratified Lie groups). We omit the details.

By Lemma 3.1, the following regularity follows for the solutions to our problem. 
Proposition 3.2 Let $\Omega$ be an arbitrary open subset of $\mathbb{R}^{N}, 0 \in \Omega$. Let $u \in \mathcal{D}_{\alpha}^{1,2}(\Omega)$ be a solution of problem (1.4) under the assumption (1.5). Then,

$$
u \in L_{l o c}^{p}(\Omega), \quad \forall p<p_{\lim }=2^{*} \frac{\sqrt{\bar{\mu}}}{\sqrt{\bar{\mu}}-\sqrt{\bar{\mu}-\mu}} .
$$

Proof By the regularity theory developed in [12,13], we can infer that $u \in \Lambda^{2}(\Omega \backslash\{0\})$. Moreover, let $R>0$ be such that $B_{R} \subset \subset \Omega$ and let $\eta \in C_{0}^{\infty}\left(B_{R}\right)$ be a cutoff function such that $\eta \equiv 1$ on $B_{R / 2}, 0 \leq \eta \leq 1$. Then, the function $w:=\eta u$ satisfies the equation

$$
-\mathcal{L}_{\alpha} w-\mu \frac{\psi^{2}}{d^{2}} w-V w+v w=g, \quad w \in \mathcal{D}_{\alpha}^{1,2}\left(B_{R}\right)
$$

where $V:=f(\xi, u) / u \in L^{Q / 2}\left(B_{R}\right)$, since $u \in L^{2^{*}}$, and $g:=-2 \nabla_{\alpha} u \cdot \nabla_{\alpha} \eta-u \mathcal{L}_{\alpha} \eta+v w$. From $\nabla_{\alpha} \eta \equiv 0$ on $B_{R / 2}(0)$ and $u \in \Lambda^{2}\left(B_{R} \backslash\{0\}\right)$, it follows that the $L^{p}$ regularity of $g$ is given by that of $w$. Therefore, starting from $g \in L^{2^{*}}$ and arguing recursively, we improve the regularity of $w$ by means of Lemma 3.1, until we reach the limitation imposed by $\mu$. The proof is therefore complete.

Remark 3.3 Note that the limit exponent for the local $L^{p}$-regularity appearing in formula (3.2) corresponds to the exponent $p_{\lim }=\frac{Q}{\sqrt{\bar{\mu}}-\sqrt{\bar{\mu}-\mu}}$, which is exactly the sharp $L^{p}$-weak summability exponent of the function $d^{-(\sqrt{\bar{\mu}}-\sqrt{\bar{\mu}-\mu})}$ appearing in the upper bound estimate (1.6). So, by means of (1.6), we shall obtain that, in fact, $u \in L_{l o c}^{\frac{Q}{\sqrt{\bar{\mu}}-\sqrt{\bar{\mu}-\mu}}, \infty}(\Omega)$.

In order to study the behavior of solutions at the singularity, we shall use a convenient transformation, following the Euclidean scheme. If $u \in \mathcal{D}_{\alpha}^{1,2}(\Omega)$ is a solution of (1.4), we define

$$
v:=d^{\beta} u, \quad \text { where } \beta=\sqrt{\bar{\mu}}-\sqrt{\bar{\mu}-\mu} .
$$

By Hardy inequality (1.3), it is easy to verify that $v \in \mathcal{D}_{\alpha}^{1,2}\left(\Omega, d^{-2 \beta} \mathrm{d} z\right)$. Moreover, using formula (2.2), we can see by direct inspection that $v$ satisfies

$$
-\operatorname{div}_{\alpha}\left(d^{-2 \beta} \nabla_{\alpha} v\right)=d^{-\beta} f\left(z, d^{-\beta} v\right), \quad z \in \Omega \backslash\{0\} .
$$

Indeed, by Eq. (1.4), we get

$$
-\left(\mathcal{L}_{\alpha} d^{-\beta}\right) v-d^{-\beta} \mathcal{L}_{\alpha} v-2\left\langle\nabla_{\alpha} d^{-\beta}, \nabla_{\alpha} v\right\rangle-\mu \frac{\psi^{2}}{d^{2}} d^{-\beta} v=f\left(z, d^{-\beta} v\right)
$$

that is, taking into account (2.2),

$$
-d^{-\beta} \mathcal{L}_{\alpha} v-2\left\langle\nabla_{\alpha} d^{-\beta}, \nabla_{\alpha} v\right\rangle-\psi^{2}\left(\beta^{2}-\beta(Q-2)+\mu\right) d^{-\beta-2}=f\left(z, d^{-\beta} v\right)
$$

which reduces to

$$
-d^{-\beta} \mathcal{L}_{\alpha} v-2\left\langle\nabla_{\alpha} d^{-\beta}, \nabla_{\alpha} v\right\rangle=f\left(z, d^{-\beta} v\right)
$$

since $\beta^{2}-\beta(Q-2)+\mu=0$ iff $\beta=\sqrt{\bar{\mu}} \pm \sqrt{\bar{\mu}-\mu}$. Then, multiplying Eq. (3.6) by $d^{-\beta}$, we obtain the equation for $v$ in (3.4).

We remark that the advantage of working with Eq. (3.4), instead of Eq. (1.4), is that, under the growth assumption (1.5) on $f$, the finite energy solutions of (3.4) turn out to be bounded, as we will prove in what follows. 
Proof of Theorem 1.1 Let $u \in \mathcal{D}_{\alpha}^{1,2}(\Omega)$ be a solution of (1.4) and define $v$ as in (3.3). Adapting the proof in [17], let $\rho>0$ be such that $B_{\rho} \subset \subset \Omega$. For $s, t>1$, let

$$
\phi:=\eta^{2} v v_{t}^{2(s-1)} \in \mathcal{D}_{\alpha}^{1,2}\left(\Omega, d^{-2 \beta} \mathrm{d} z\right),
$$

where $v_{t}=\min \{|v|, t\}, \eta \in C_{0}^{\infty}\left(B_{\rho}\right), 0 \leq \eta \leq 1, \eta \equiv 1$ in $B_{r}$, with $0<r<\rho$ and $\left|\nabla_{\alpha} \eta\right| \leq \frac{4}{\rho-r}$. By using $\phi$ as a test function in (3.4), we get

$$
\begin{aligned}
& \int_{\Omega} d^{-2 \beta}\left(2 \eta v v_{t}^{2(s-1)}\left\langle\nabla_{\alpha} \eta, \nabla_{\alpha} v\right\rangle+\eta^{2} v_{t}^{2(s-1)}\left|\nabla_{\alpha} v\right|^{2}+2(s-1) \eta^{2} v_{t}^{2(s-1)}\left|\nabla_{\alpha} v_{t}\right|^{2}\right) \mathrm{d} z \\
& \quad=\int_{\Omega} d^{-\beta} f\left(z, d^{\beta} v\right) \eta^{2} v v_{t}^{2(s-1)} \mathrm{d} z .
\end{aligned}
$$

For any $\varepsilon>0$ small, it holds

$$
\begin{aligned}
\left|\int_{\Omega} 2 d^{-2 \beta} \eta v v_{t}^{2(s-1)}\left\langle\nabla_{\alpha} \eta, \nabla_{\alpha} v\right\rangle \mathrm{d} z\right| \leq & \varepsilon \int_{\Omega} d^{-2 \beta} \eta^{2} v_{t}^{2(s-1)}\left|\nabla_{\alpha} v\right|^{2} \mathrm{~d} z \\
& +C(\varepsilon) \int_{\Omega} d^{-2 \beta}|v|^{2} v_{t}^{2(s-1)}\left|\nabla_{\alpha} \eta\right|^{2} \mathrm{~d} z
\end{aligned}
$$

Then, by (3.7) and (3.8), by choosing $\varepsilon$ sufficiently small and using the growth assumptions (1.5) on $f$, we get

$$
\begin{aligned}
& \int_{\Omega} d^{-2 \beta}\left(\eta^{2} v_{t}^{2(s-1)}\left|\nabla_{\alpha} v\right|^{2}+2(s-1) \eta^{2} v_{t}^{2(s-1)}\left|\nabla_{\alpha} v_{t}\right|^{2}\right) \mathrm{d} z \\
& \leq C \int_{\Omega} d^{-2 \beta}\left(\eta^{2}+\left|\nabla_{\alpha} \eta\right|^{2}\right)|v|^{2} v_{t}^{2(s-1)} \mathrm{d} z \\
& \quad+C \int_{\Omega} d^{-2^{*} \beta} \eta^{2}|v|^{2^{*}} v_{t}^{2(s-1)} \mathrm{d} z .
\end{aligned}
$$

Now, consider the following weighted Sobolev inequality for the Grushin gradient

$$
\left(\int_{\Omega} d^{-2^{*} \beta}|w|^{2^{*}} \mathrm{~d} z\right)^{\frac{2}{2^{*}}} \leq C \int_{\Omega} d^{-2 \beta}\left|\nabla_{\alpha} w\right|^{2} \mathrm{~d} z, \quad \forall w \in \mathcal{D}_{\alpha}^{1,2}\left(\Omega, d^{-2 \beta} \mathrm{d} z\right)
$$

which follows from the general weighted Hardy-Sobolev inequality recalled in Sect. 2, formula (2.6). Taking $w=\eta v v_{t}^{s-1}$ in (3.10) and using (3.9), we obtain

$$
\begin{aligned}
& \left(\int_{\Omega} d^{-2^{*} \beta}\left|\eta v v_{t}^{s-1}\right|^{2^{*}} \mathrm{~d} z\right)^{\frac{2}{2^{*}}} \\
& \leq C \int_{\Omega} d^{-2 \beta}\left|\nabla_{\alpha}\left(\eta v v_{t}^{s-1}\right)\right|^{2} \mathrm{~d} z \\
& \leq C \int_{\Omega} d^{-2 \beta}\left(\left|\nabla_{\alpha} \eta\right|^{2}|v|^{2} v_{t}^{2(s-1)}+\eta^{2} v_{t}^{2(s-1)}\left|\nabla_{\alpha} v\right|^{2}+(s-1)^{2} \eta^{2} v_{t}^{2(s-1)}\left|\nabla_{\alpha} v_{t}\right|^{2}\right) \mathrm{d} z \\
& \leq C s \int_{\Omega} d^{-2 \beta}\left(\eta^{2}+\left|\nabla_{\alpha} \eta\right|^{2}\right)|v|^{2} v_{t}^{2(s-1)} \mathrm{d} z+C s \int_{\Omega} d^{-2^{*} \beta} \eta^{2}|v|^{2^{*}} v_{t}^{2(s-1)} \mathrm{d} z .
\end{aligned}
$$

Since $u \in L_{l o c}^{p}(\Omega)$ for any $p<\frac{2 Q}{Q-2-2(\sqrt{\bar{\mu}-\mu})}$ (see Proposition 3.2), we choose

$$
\frac{Q}{2}<q<\frac{Q(Q-2)}{2(Q-2-2 \sqrt{\bar{\mu}-\mu})}
$$


so that

$$
\left(2^{*}-2\right) q<\frac{2 Q}{Q-2-2(\sqrt{\bar{\mu}-\mu})} \text { and } 2<\frac{2 q}{q-1}<2^{*} .
$$

By this choice and reasoning as in [17], the last integral in (3.11) can be estimated as follows, for any $\varepsilon>0$

$$
\begin{aligned}
& \int_{\Omega} d^{-2^{*} \beta} \eta^{2}|v|^{2^{*}} v_{t}^{2(s-1)} \mathrm{d} z \\
& \quad=\int_{\Omega} d^{-2 \beta}|u|^{2^{*}-2}\left|\eta v v_{t}^{s-1}\right|^{2} \mathrm{~d} z \\
& \quad \leq\|u\|_{L^{\left(2^{*}-2\right) q}(\text { supp } \eta)}^{2^{*}-2}\left\|d^{\beta} \eta v v_{t}^{s-1}\right\|^{2} L^{\frac{2 q}{q-1}}(\Omega) \\
& \quad \leq C \varepsilon^{2}\left(\int_{\Omega} d^{-2^{*} \beta}\left|\eta v v_{t}^{s-1}\right|^{2^{*}} \mathrm{~d} z\right)^{\frac{2}{2^{*}}}+C \varepsilon^{-\frac{2 Q}{2 q-Q}} \int_{\Omega} d^{-2 \beta}\left|\eta v v_{t}^{s-1}\right|^{2} \mathrm{~d} z
\end{aligned}
$$

So, by (3.11) and (3.12), we have

$$
\begin{aligned}
\left(\int_{\Omega} d^{-2^{*} \beta}\left|\eta v v_{t}^{s-1}\right|^{2^{*}} \mathrm{~d} z\right)^{\frac{2}{2^{*}}} \leq & C s \varepsilon^{2}\left(\int_{\Omega} d^{-2^{*} \beta}\left|\eta v v_{t}^{s-1}\right|^{2^{*}} \mathrm{~d} z\right)^{\frac{2}{2^{*}}} \\
& +C s \int_{\Omega} d^{-2 \beta}\left(\eta^{2}+\left|\nabla_{\alpha} \eta\right|^{2}\right)|v|^{2} v_{t}^{2(s-1)} \mathrm{d} z \\
& +C s \varepsilon^{-\frac{2 Q}{2 q-Q}} \int_{\Omega} d^{-2 \beta}\left|\eta v v_{t}^{s-1}\right|^{2} \mathrm{~d} z .
\end{aligned}
$$

Taking $\varepsilon=\frac{1}{\sqrt{2 C s}}$ in (3.13), we obtain

$$
\left(\int_{\Omega} d^{-2^{*} \beta}\left|\eta v v_{t}^{s-1}\right|^{2^{*}} \mathrm{~d} z\right)^{\frac{2}{2^{*}}} \leq C s^{\alpha} \int_{\Omega} d^{-2 \beta}\left(\eta^{2}+\left|\nabla_{\alpha} \eta\right|^{2}\right)|v|^{2} v_{t}^{2(s-1)} \mathrm{d} z,
$$

where $\alpha=\frac{2 q}{2 q-Q}>0$. Note that

$$
\int_{\Omega} d^{-2^{*} \beta} \eta^{2^{*}}|v|^{2} v_{t}^{2^{*} s-2} \mathrm{~d} z \leq \int_{\Omega} d^{-2^{*} \beta}\left|\eta v v_{t}^{s-1}\right|^{2^{*}} \mathrm{~d} z .
$$

Hence, from (3.15) and (3.14), we have

$$
\begin{aligned}
& \left(\int_{\Omega} d^{-2^{*} \beta} \eta^{2^{*}}|v|^{2} v_{t}^{2^{*} s-2} \mathrm{~d} z\right)^{\frac{2}{2^{*}}} \\
& \leq C s^{\alpha} \int_{\Omega} d^{-2 \beta}\left(\eta^{2}+\left|\nabla_{\alpha} \eta\right|^{2}\right)|v|^{2} v_{t}^{2(s-1)} \mathrm{d} z \\
& \leq C s^{\alpha} \int_{\Omega} d^{-2^{*} \beta}\left(\eta^{2}+\left|\nabla_{\alpha} \eta\right|^{2}\right)|v|^{2} v_{t}^{2(s-1)} \mathrm{d} z .
\end{aligned}
$$

By the definition of $\eta$, we then obtain

$$
\left(\int_{B_{r}} d^{-2^{*} \beta}|v|^{2} v_{t}^{2^{*} s-2} \mathrm{~d} z\right)^{\frac{2}{2^{*}}} \leq \frac{C s^{\alpha}}{(\rho-r)^{2}} \int_{B_{\rho}} d^{-2^{*} \beta}|v|^{2} v_{t}^{2 s-2} \mathrm{~d} z .
$$

Choosing $s^{*}$ such that

$$
\frac{Q}{Q-2}<s^{*}<\frac{Q}{Q-2-2 \sqrt{\bar{\mu}-\mu}}
$$


we consider the sequence $s_{j}=s^{*}\left(\frac{2^{*}}{2}\right)^{j}, j=0,1,2, \ldots$ Let $\rho_{0}$ be sufficiently small that $B_{2 \rho_{0}} \subset \subset \Omega$ and let $r_{j}=\rho_{0}\left(1+\rho_{0}^{j}\right), j=0,1,2, \ldots$ Taking $\rho=r_{j}, r=r_{j+1}$ and $s=s_{j}$ in (3.16), by means of Moser's iteration technique, we finally obtain

$$
\begin{aligned}
& \left(\int_{B_{r_{j+1}}} d^{-2^{*} \beta}|v|^{2} v_{t}^{2 s_{j+1}-2} \mathrm{~d} z\right)^{\frac{1}{2 s_{j+1}}} \\
& \leq\left(\frac{C}{\left(1-\rho_{0}\right) \rho_{0}}\right)^{\sum_{k=0}^{j} \frac{1}{2 s_{k}}} \rho_{0}^{-\sum_{k=0}^{j} \frac{k}{2 s_{k}}} \prod_{k=0}^{j} s_{k}^{\frac{\alpha}{2 s_{k}}}\left(\int_{B_{r_{0}}} d^{-2^{*} \beta}|v|^{2} v_{t}^{2 s^{*}-2} \mathrm{~d} z\right)^{\frac{1}{2 s^{*}}},
\end{aligned}
$$

where the integral in the right-hand side of (3.17) if finite, since

$$
\int_{B_{r_{0}}} d^{-2^{*} \beta}|v|^{2} v_{t}^{2 s^{*}-2} \mathrm{~d} z \leq r_{0}^{\left(2 s^{*}-2^{*}\right) \beta} \int_{B_{r_{0}}}|u|^{2 s^{*}} \mathrm{~d} z<\infty .
$$

So, letting $j \rightarrow \infty$ in (3.17), we get that

$$
\left\|v_{t}\right\|_{L^{\infty}\left(B_{\rho_{0}}\right)} \leq C
$$

with $C$ independent of $t$. Therefore, letting $t \rightarrow+\infty$, we obtain that $v$ is bounded on $B_{\rho_{0}}$, that is equivalent to the upper bound (1.6).

To prove the lower bound (1.7), let $u$ be a positive solution of problem (1.4) and suppose that $f(z, t) \geq 0$ for $t>0$. As before, let $v$ be defined as in (3.3). We follow the proof in CaoHan [3, Theorem 1.1]. Let $0<\rho_{1}<\rho_{2}$ such that $B_{\rho_{2}} \subset \subset \Omega$ and $\operatorname{set} \varphi(\rho):=\min _{d(z)=\rho} v(z)$, $\rho_{1}<\rho<\rho_{2}$. Consider the comparison function

$$
g(z):=A d(z)^{-2 \sqrt{\bar{\mu}-\mu}}+B,
$$

where the constants $A$ and $B$ are chosen so that $g(z)=\varphi\left(\rho_{i}\right)$ for $d(z)=\rho_{i}$, for $i=1,2$. The explicit expression of $A$ and $B$ turns out to be

$$
A=\frac{\varphi\left(\rho_{2}\right)-\varphi\left(\rho_{1}\right)}{\rho_{2}^{-2 \sqrt{\bar{\mu}-\mu}}-\rho_{1}^{-2 \sqrt{\bar{\mu}-\mu}}}, \quad B=\frac{\varphi\left(\rho_{1}\right) \rho_{2}^{-2 \sqrt{\bar{\mu}-\mu}}-\varphi\left(\rho_{2}\right) \rho_{1}^{-2 \sqrt{\bar{\mu}-\mu}}}{\rho_{2}^{-2 \sqrt{\bar{\mu}-\mu}}-\rho_{1}^{-2 \sqrt{\bar{\mu}-\mu}}} .
$$

By direct calculation, it is easy to verify that

$$
\operatorname{div}_{\alpha}\left(d^{-2 \beta} \nabla_{\alpha} g\right)=0, \quad \forall z \neq 0 .
$$

So, taking into account (3.4) and the sign hypothesis on $f$, it follows that

$$
-\operatorname{div}_{\alpha}\left(d^{-2 \beta} \nabla_{\alpha}(v-g)\right) \geq 0 \text { in } \Omega \backslash\{0\} .
$$

On the other hand, by the definition of $g$, we know that $v \geq g$ on $\partial\left(B_{\rho_{2}} \backslash B_{\rho_{1}}\right)$. Therefore, by the weak maximum principle, we obtain that

$$
v \geq g \quad \text { in } B_{\rho_{2}} \backslash B_{\rho_{1}} .
$$


So, taking into account the explicit form of $g$, from (3.18), we get

$$
\begin{aligned}
& v(z) \geq \frac{\varphi\left(\rho_{2}\right)-\varphi\left(\rho_{1}\right)}{\rho_{2}^{-2 \sqrt{\mu-\mu}}-\rho_{1}^{-2 \sqrt{\bar{\mu}-\mu}}} d(z)^{-2 \sqrt{\mu}-\mu}+\frac{\varphi\left(\rho_{1}\right) \rho_{2}^{-2 \sqrt{\mu}-\mu}-\varphi\left(\rho_{2}\right) \rho_{1}^{-2 \sqrt{\mu}-\mu}}{\rho_{2}^{-2 \sqrt{\mu-\mu}}-\rho_{1}^{-2 \sqrt{\bar{\mu}-\mu}}}
\end{aligned}
$$

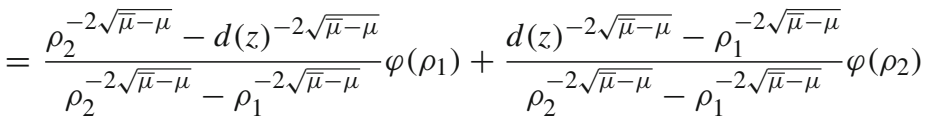

$$
\begin{aligned}
& \geq \frac{d(z)^{2 \sqrt{\mu}-\mu}-\rho_{1}^{2 \sqrt{\mu}-\mu}}{d(z)^{2 \sqrt{\bar{\mu}-\mu}}\left(1-\rho_{1}^{2 \sqrt{\bar{\mu}-\mu}} \rho_{2}^{-2 \sqrt{\bar{\mu}-\mu}}\right)} \varphi\left(\rho_{2}\right),
\end{aligned}
$$

for every $z \in B_{\rho_{2}} \backslash B_{\rho_{1}}$.

Finally, letting $\rho_{1} \rightarrow 0$ in (3.19), we obtain that $v(z) \geq \varphi\left(\rho_{2}\right)=\min _{d(\eta)=\rho_{2}} v(\eta)>0$ for all $z \in B_{\rho_{2}} \backslash\{0\}$, that is, (1.7).

\section{Asymptotic behavior at infinity}

This section is devoted to study the asymptotic behavior at infinity of solutions to problem (1.2) on exterior domains of $\mathbb{R}^{N}$.

Due to the conformal invariance of the critical Eq. (1.2), the rate of decay of solutions at infinity can be directly deduced from their asymptotic behavior at 0 . In the Euclidean case, this is achieved by means of the classical Kelvin transform on $\mathbb{R}^{N}$ (see, e.g., [8]). In our framework, we shall use the Kelvin-type transform constructed on the geometry of Grushin vector fields, introduced by Monti and Morbidelli in [33].

In order to prove the asymptotic decay result, we briefly recall the definition and the main properties of such Kelvin transform. Using the dilations (1.11), a spherical inversion $\sigma$ constructed on the Grushin geometry is defined on $\mathbb{R}^{N}$ by

$$
\sigma(z)=\delta_{d(z)^{-2}}(z), \quad z \neq 0 .
$$

A direct calculation (see [33, Lemma 2]) shows that the Jacobian of $\sigma$ satisfies

$$
\left|J_{\sigma}(z)\right|=d(z)^{-2 Q}=\Gamma(z)^{\frac{2 Q}{Q-2}}, \quad \forall z \neq 0 .
$$

The inversion $\sigma$ is a conformal map for the Grushin gradient in the following sense

$$
\left|\nabla_{\alpha}(u \circ \sigma)(z)\right|^{2}=\left|J_{\sigma}(z)\right|^{2 / Q}\left|\left(\nabla_{\alpha} u\right)(\sigma(z))\right|^{2} \quad \forall u \in C^{1}\left(\mathbb{R}^{N}\right), z \neq 0 .
$$

By means of $\sigma$, the following Kelvin-type transform can be defined. Given a function $u$ : $\mathbb{R}^{N} \rightarrow \mathbb{R}$, we define $u^{*}: \mathbb{R}^{N} \backslash\{0\} \rightarrow \mathbb{R}$ as the function

$$
u^{*}(z)=\Gamma(z) u(\sigma(z)), \quad z \neq 0 .
$$

The function $u^{*}$ has, a priori, a singularity at $z=0$. This singularity, however, turns out to be removable, as proved in [33].

Using the conformal property (4.2), it can be proved that the Kelvin transform $u^{*}$ preserves the critical equation with Hardy perturbation (1.2), as we will verify below as a consequence of Theorem 4.1.

In what follows, we shall denote by $\Omega^{*}$ the image of a generic domain $\Omega \subset \mathbb{R}^{N}$ under the inversion $\sigma$. Note that, if $\Omega$ is a neighborhood of $\infty$, i.e., there exists a ball $B_{R}$ such that 
$\bar{B}_{R}^{C} \subset \Omega$, then $\Omega^{*}$ is a punctured neighborhood of 0 , i.e., $\Omega^{*}=D \backslash\{0\}$, where $D$ is an open set, $0 \in D$.

For $\mu \in\left[0, \bar{\mu}\left[\right.\right.$, consider the following scalar product on $\mathcal{D}_{\alpha}^{1,2}(\Omega)$ :

$$
\langle u, v\rangle_{\mu}:=\left(\int_{\Omega}\left\langle\nabla_{\alpha} u, \nabla_{\alpha} v\right\rangle \mathrm{d} z-\mu \int_{\Omega} \psi^{2} \frac{u v}{d^{2}} \mathrm{~d} z\right)^{1 / 2}
$$

Observe that the norm induced by $\langle\cdot, \cdot\rangle_{\mu}$ is equivalent to the Dirichlet norm $\|u\|=$ $\left(\int_{\Omega}\left|\nabla_{\alpha} u\right|^{2} \mathrm{~d} z\right)^{1 / 2}$, due to Hardy inequality (1.3). The following property holds.

Theorem 4.1 The Kelvin transform defined in (4.3) is an isometry between $\mathcal{D}_{\alpha}^{1,2}(\Omega)$ and $\mathcal{D}_{\alpha}^{1,2}\left(\Omega^{*}\right)$ with respect to the scalar product (4.4).

Proof Let $u, v \in \mathcal{D}_{\alpha}^{1,2}(\Omega)$ and let $u^{*}, v^{*}$ their Kelvin transform. As proved in [33, Theorem 2.5], it holds that

$$
\int_{\Omega}\left\langle\nabla_{\alpha} u, \nabla_{\alpha} v\right\rangle \mathrm{d} z=\int_{\Omega^{*}}\left\langle\nabla_{\alpha} u^{*}, \nabla_{\alpha} v^{*}\right\rangle \mathrm{d} z .
$$

Concerning the Hardy term, we have that

$$
\begin{aligned}
\int_{\Omega^{*}} \psi^{2} \frac{u^{*} v^{*}}{d^{2}} \mathrm{~d} z & =\int_{\Omega^{*}} \psi^{2} d^{2(2-Q)} \frac{u(\sigma(z)) v(\sigma(z))}{d^{2}} \mathrm{~d} z \\
& =\int_{\Omega} \psi^{2} \frac{u v}{d^{2}} \mathrm{~d} z
\end{aligned}
$$

where we have used that $d(\sigma(z))=d(z)^{-1}$ for any $z \neq 0$ and that, since $\psi$ is homogeneous of degree 0 with respect to the dilations $\delta_{\lambda}$, then $\psi(\sigma(z))=\psi(z)$, for all $z \in \mathbb{R}^{N} \backslash\{0\}$. The proof is therefore complete.

Lemma 4.2 If $u \in \mathcal{D}_{\alpha}^{1,2}(\Omega)$ is a solution to

$$
-\mathcal{L}_{\alpha} u-\mu \frac{\psi^{2}}{d^{2}} u=f(z, u) \text { in } \Omega,
$$

then $u^{*} \in \mathcal{D}_{\alpha}^{o}, 2\left(\Omega^{*}\right)$ satisfies

$$
-\mathcal{L}_{\oslash} u^{*}-\mu \frac{\psi^{2}}{d^{2}} u^{*}=\frac{1}{d^{Q+2}} f\left(\sigma(z), d^{Q-2} u^{*}\right) \text { in } \Omega^{*}
$$

Proof Let $u \in \mathcal{D}_{\alpha}^{1,2}(\Omega)$ be a solution to (4.5). By Theorem 4.1, we know that $u^{*} \in \mathcal{D}_{\alpha}^{1,2}\left(\Omega^{*}\right)$. Let $\varphi \in C_{0}^{\infty}\left(\Omega^{*}\right)$; then, we can write $\varphi=\phi^{*}$, for some $\phi \in C_{0}^{\infty}(\Omega)$. Applying Theorem 4.1 and property (4.1), we have 


$$
\begin{aligned}
\int_{\Omega^{*}} & \left\langle\nabla_{\alpha} u^{*}, \nabla_{\alpha} \varphi\right\rangle \mathrm{d} z-\mu \int_{\Omega^{*}} \psi^{2} \frac{u^{*} \varphi}{d^{2}} \mathrm{~d} z \\
= & \int_{\Omega^{*}}\left\langle\nabla_{\alpha} u^{*}, \nabla_{\alpha} \phi^{*}\right\rangle \mathrm{d} z-\mu \int_{\Omega^{*}} \psi^{2} \frac{u^{*} \phi^{*}}{d^{2}} \mathrm{~d} z \\
= & \int_{\Omega}\left\langle\nabla_{\alpha} u, \nabla_{\alpha} \phi\right\rangle \mathrm{d} z-\mu \int_{\Omega} \psi^{2} \frac{u \phi}{d^{2}} \mathrm{~d} z \\
= & \int_{\Omega} f(z, u) \phi \mathrm{d} z \\
= & \int_{\Omega^{*}} f(\sigma(z), u(\sigma(z))) \phi(\sigma(z))\left|J_{\sigma}(z)\right| \mathrm{d} z \\
= & \int_{\Omega^{*}} d^{-2-Q} f\left(\sigma(z), d^{Q-2} u^{*}\right) \phi^{*} \mathrm{~d} z \\
= & \int_{\Omega^{*}} d^{-2-Q} f\left(\sigma(z), d^{Q-2} u^{*}\right) \varphi \mathrm{d} z .
\end{aligned}
$$

By the arbitrariness of $\varphi \in C_{0}^{\infty}\left(\Omega^{*}\right)$, the thesis follows.

Proof of Theorem 1.2 Let $u$ be a nonnegative solution of Eq. (1.8). The behavior at the origin (1.9) directly follows from Theorem 1.1. To prove the decay estimate at infinity (1.10), observe that, by Lemma 4.2, if $u$ satisfies the critical Eq. (1.8) in a neighborhood of infinity, then $u^{*}$ satisfies the same critical equation in a neighborhood of the origin, where $K(z)$ is substituted by $K(\sigma(z))$, i.e.,

$$
-\mathcal{L}_{\alpha} u^{*}-\mu \frac{\psi^{2}}{d^{2}} u^{*}=K(\sigma(z))\left(u^{*}\right)^{2^{*}-1} \text { in } \Omega^{*} .
$$

So, by Theorem 1.1,

$$
u^{*}(z) \sim d(z)^{-\sqrt{\bar{\mu}}+\sqrt{\bar{\mu}-\mu}} \text { for } d(z) \text { small. }
$$

Hence, taking into account that $u(z)=d(z)^{2-Q} u^{*}(\sigma(z))$, the decay estimate at infinity (1.10) follows.

\section{Some integral identities and nonexistence results}

In this section, by means of Pohozaev-type arguments [35], we prove some nonexistence results for positive solutions to problem (1.2). We recall that Pohozaev-type identities in the subelliptic setting have been extensively studied, starting from the seminal paper by Garofalo and Lanconelli [15], and they present several additional difficulties with respect to the Euclidean framework. We shall refer, in particular, to [20,27] for their relation to the present results.

In order to treat our critical problem with Hardy perturbation (1.2), as in the previous steps, it will be convenient to use the transformation $v=d^{\beta} u, \beta=\sqrt{\bar{\mu}}-\sqrt{\bar{\mu}-\mu}$, introduced in (3.3), being $u$ a weak solution of (1.2). So, taking into account (3.4), the original problem (1.2) turns into the equivalent one

$$
-\operatorname{div}_{\alpha}\left(d^{-2 \beta} \nabla_{\alpha} v\right)=K(z) \frac{|v|^{2^{*}-2} v}{d^{2^{*} \beta}} \text { in } \Omega .
$$

As previously observed, the advantage of working with Eq. (5.1) is that its solutions are bounded at 0 , as proved in Sect. 3 . For a refined analysis of the regularity at 0 of solutions 
to equations like (5.1) in the nondegenerate case $\alpha=0$ we quote [9], where it is proved that weak solutions of such problems are, in fact, Hölder continuous at 0 .

The following Pohozaev-type identity for solutions to Eq. (5.1) holds. The Euclidean counterpart is given by Theorem 2.1 in Felli-Schneider [10]; the identity in the Grushin non-singular case $\beta=0$ with autonomous nonlinearities is contained in [20].

Theorem 5.1 Let $\Omega \subset \mathbb{R}^{N}$ be a bounded domain with $C^{1}$-boundary, $0 \in \Omega, K \in C^{1}(\bar{\Omega})$ and $v \in S_{\alpha, \beta}^{1,2}(\Omega) \cap \Lambda^{2}(\bar{\Omega} \backslash\{0\})$ be a solution of $E q$. (5.1). Then, the following identity holds

$$
\begin{aligned}
\frac{1}{2^{*}} & \int_{\Omega} Z K \frac{|v|^{2^{*}}}{d^{2^{*} \beta}} \mathrm{d} z-\frac{1}{2^{*}} \int_{\partial \Omega} K(z) \frac{|v|^{2^{*}}}{d^{2^{*} \beta}}\langle Z, v\rangle \mathrm{d} \sigma \\
= & \frac{Q-2-2 \beta}{2} \int_{\partial \Omega} d^{-2 \beta} v\left\langle\nabla_{\alpha} v, v_{\alpha}\right\rangle \mathrm{d} \sigma-\frac{1}{2} \int_{\partial \Omega} d^{-2 \beta}\left|\nabla_{\alpha} v\right|^{2}\langle Z, v\rangle \mathrm{d} \sigma \\
& +\int_{\partial \Omega} d^{-2 \beta}\left\langle\nabla_{\alpha} v, v_{\alpha}\right\rangle Z v \mathrm{~d} \sigma
\end{aligned}
$$

where $Z$ is the infinitesimal generator of the dilations $\delta_{\lambda}$ defined in $(2.4), v=\left(v_{1}, \ldots, v_{N}\right)$ is the outward unit normal to $\partial \Omega$ and $v_{\alpha}=\left(v_{1}, \ldots, v_{m},|x|^{\alpha} v_{m+1}, \ldots,|x|^{\alpha} v_{N}\right)$.

Proof We first prove the theorem by assuming $v \in C^{2}(\bar{\Omega} \backslash\{0\})$. The proof can be completed for $v \in \Lambda^{2}(\bar{\Omega} \backslash\{0\})$ by means of an approximation argument, arguing as in [20, page 4643]. We shall omit the details.

Due to the lack of regularity at the origin, in order to prove (5.2), we consider approximating domains $\Omega \backslash B_{r_{n}}$, for an appropriate sequence of radii $r_{n} \rightarrow 0$, as in [10,27]. To this aim, observe that, from Federer's coarea formula (see [7]), if $B_{R}=B_{d}(0, R)$ is a $d$-ball centered at 0 contained in $\Omega$, then

$$
\int_{0}^{R} \mathrm{~d} s \int_{\partial B_{S}}\left(K(z) \frac{|v|^{2^{*}}}{d^{2^{*} \beta}}+\frac{\left|\nabla_{\alpha} v\right|^{2}}{d^{2 \beta}}\right) \frac{1}{|\nabla d|} \mathrm{d} \sigma=\int_{B_{R}}\left(K(z) \frac{|v|^{2^{*}}}{d^{2^{*} \beta}}+\frac{\left|\nabla_{\alpha} v\right|^{2}}{d^{2 \beta}}\right) \mathrm{d} z .
$$

Now, since $v \in S_{\alpha, \beta}^{1,2}(\Omega)$ (see the definition in Sect. 2), the integral in the right-hand side of (5.3) is finite. This implies that there exists a sequence $r_{n} \rightarrow 0$ such that

$$
r_{n} \int_{\partial B_{r_{n}}}\left(K(z) \frac{|v|^{2^{*}}}{d^{2^{*} \beta}}+\frac{\left|\nabla_{\alpha} v\right|^{2}}{d^{2 \beta}}\right) \frac{1}{|\nabla d|} \mathrm{d} \sigma \longrightarrow 0, \text { as } n \rightarrow \infty .
$$

Let $\Omega_{r_{n}}:=\Omega \backslash B_{r_{n}}$. Multiplying Eq. (5.1) by $Z v$ and integrating over $\Omega_{r_{n}}$, we get

$$
\int_{\Omega_{r_{n}}}-\operatorname{div}_{\alpha}\left(d^{-2 \beta} \nabla_{\alpha} v\right) Z v \mathrm{~d} z=\int_{\Omega_{r_{n}}} K(z) \frac{|v|^{2^{*}-2} v}{d^{2^{*} \beta}} Z v \mathrm{~d} z .
$$

Concerning the left-hand side of (5.5), we claim that the following Rellich-type identity holds, which represents a weighted version of the identity in [14, Theorem 2.2] or its generalization to $\Delta_{\lambda}$-operators in [20, Theorem 2.1]:

$$
\begin{gathered}
\int_{\Omega_{r_{n}}} \operatorname{div}_{\alpha}\left(d^{-2 \beta} \nabla_{\alpha} v\right) Z v \mathrm{~d} z=\frac{Q-2-2 \beta}{2} \int_{\Omega_{r_{n}}} d^{-2 \beta}\left|\nabla_{\alpha} v\right|^{2} \mathrm{~d} z \\
-\frac{1}{2} \int_{\partial \Omega_{r_{n}}} d^{-2 \beta}\left|\nabla_{\alpha} v\right|^{2}\langle Z, v\rangle \mathrm{d} \sigma+\int_{\partial \Omega_{r_{n}}} d^{-2 \beta}\left\langle\nabla_{\alpha} v, v_{\alpha}\right\rangle Z v \mathrm{~d} \sigma .
\end{gathered}
$$


To prove it, we use the general integral identity proved in Garofalo-Lanconelli [15], which we here recall. Let $D \subset \mathbb{R}^{N}$ be a bounded piecewise $C^{1}$-domain, let $C=\left(c_{i j}\right)$ be a $N \times N$ symmetric matrix with $C^{1}$-entries and consider the operator $\operatorname{div}(C \nabla)$ in $\mathbb{R}^{N}$; let $X$ be a smooth vector field in $\mathbb{R}^{N}$ with components $X_{1}, \ldots, X_{N}$. Moreover, denote by $v_{, i}$ the partial derivative $\partial v / \partial z_{i}$ and let $c_{i j, k}$ denote $\partial c_{i j} / \partial z_{k}$. The following identity holds

$$
\begin{aligned}
\int_{D} \operatorname{div}(C \nabla v) X v \mathrm{~d} z= & \frac{1}{2} \int_{D} \operatorname{div} X\langle C \nabla v, \nabla v\rangle \mathrm{d} z-\int_{D} \partial X_{i} / \partial z_{j}(C \nabla v)_{j} v_{, i} \mathrm{~d} z \\
& +\frac{1}{2} \int_{D} X_{i} c_{j k, i} v_{, k} v_{, j} \mathrm{~d} z-\frac{1}{2} \int_{\partial D}\langle C \nabla v, \nabla v\rangle\langle X, v\rangle \mathrm{d} \sigma \\
& +\int_{\partial D}\langle C \nabla v, v\rangle X v \mathrm{~d} \sigma .
\end{aligned}
$$

Note that the operator $P_{\alpha, \beta}:=\operatorname{div}_{\alpha}\left(d^{-2 \beta} \nabla_{\alpha}\right)$ which appears in the left-hand side of (5.6) can be expressed in divergence form as

$$
P_{\alpha, \beta}=\operatorname{div}\left(d^{-2 \beta} A_{\alpha} \nabla\right)
$$

where "div" is the Euclidean divergence on $\mathbb{R}^{N}$ and $A_{\alpha}$ is the matrix defined in (2.1). Now, following [14, proof of Theorem 2.2], we define the approximating matrices

$$
A_{\alpha}^{\varepsilon}=\left(\begin{array}{c|c}
I_{\mathbb{R}^{m}} & 0 \\
\hline 0 & \left(|x|^{2}+\varepsilon^{2}\right)^{\alpha} I_{\mathbb{R}^{n}}
\end{array}\right)
$$

Observe that the matrix $d^{-2 \beta} A_{\alpha}^{\varepsilon}$ has smooth entries in domains not containing the origin, so we can apply (5.7) on $D=\Omega_{r_{n}}$ with $C=d^{-2 \beta} A_{\alpha}^{\varepsilon}$ and $X=Z$. By the definition of $Z$ (see (2.4)), we get that

$$
\left(\partial Z_{i} / \partial z_{j}\right)=\operatorname{diag}[\underbrace{1, \ldots, 1}_{m}, \underbrace{\alpha+1, \ldots, \alpha+1}_{n}],
$$

so that

$$
\begin{aligned}
\partial Z_{i} / \partial z_{j}\left(d^{-2 \beta} A_{\alpha}^{\varepsilon} \nabla v\right)_{j} v_{, i} & =d^{-2 \beta}\left(\left|\nabla_{x} v\right|^{2}+(\alpha+1)\left(|x|^{2}+\varepsilon^{2}\right)^{\alpha}\left|\nabla_{y} v\right|^{2}\right) \\
& =d^{-2 \beta}\left(\left\langle A_{\alpha}^{\varepsilon} \nabla v, \nabla v\right\rangle+\alpha\left(|x|^{2}+\varepsilon^{2}\right)^{\alpha}\left|\nabla_{y} v\right|^{2}\right) .
\end{aligned}
$$

Moreover,

$$
\begin{aligned}
Z_{i}\left(d^{-2 \beta} A_{\alpha}^{\varepsilon}\right)_{j k, i} v_{, k} v_{, j}= & -2 \beta d^{-2 \beta-1}\langle Z, \nabla d\rangle\left(A_{\alpha}^{\varepsilon}\right)_{j k} v_{, k} v_{, j} \\
& +2 d^{-2 \beta} \alpha|x|^{2}\left(|x|^{2}+\varepsilon^{2}\right)^{\alpha-1}\left|\nabla_{y} v\right|^{2} \\
= & -2 \beta d^{-2 \beta}\left\langle A_{\alpha}^{\varepsilon} \nabla v, \nabla v\right\rangle+2 d^{-2 \beta} \alpha|x|^{2}\left(|x|^{2}+\varepsilon^{2}\right)^{\alpha-1}\left|\nabla_{y} v\right|^{2}
\end{aligned}
$$

where we have used (2.5). Now, inserting (5.8) and (5.9) into (5.7) and taking into account that $\operatorname{div} Z=Q$, we get

$$
\begin{aligned}
& \int_{\Omega_{r_{n}}} \operatorname{div}\left(d^{-2 \beta} A_{\alpha}^{\varepsilon} \nabla v\right) Z v \mathrm{~d} z=\frac{Q-2-2 \beta}{2} \int_{\Omega_{r_{n}}} d^{-2 \beta}\left\langle A_{\alpha}^{\varepsilon} \nabla v, \nabla v\right\rangle \mathrm{d} z \\
& -\alpha \int_{\Omega_{r_{n}}} d^{-2 \beta} \frac{\varepsilon^{2}}{|x|^{2}+\varepsilon^{2}}\left(|x|^{2}+\varepsilon^{2}\right)^{\alpha}\left|\nabla_{y} v\right|^{2} \mathrm{~d} z \\
& -\frac{1}{2} \int_{\partial \Omega_{r_{n}}} d^{-2 \beta}\left\langle A_{\alpha}^{\varepsilon} \nabla v, \nabla v\right\rangle\langle Z, v\rangle \mathrm{d} \sigma+\int_{\partial \Omega_{r_{n}}} d^{-2 \beta}\left\langle A_{\alpha}^{\varepsilon} \nabla v, v\right\rangle Z v \mathrm{~d} \sigma .
\end{aligned}
$$


Hence, letting $\varepsilon \rightarrow 0$ in (5.10) and taking into account that $\left\langle A_{\alpha} \nabla v, \nabla v\right\rangle=\left\langle\nabla_{\alpha} v, \nabla_{\alpha} v\right\rangle$ and $\left\langle A_{\alpha} \nabla v, v\right\rangle=\left\langle\nabla_{\alpha} v, v_{\alpha}\right\rangle$, the claim (5.6) follows.

Concerning the right-hand side of (5.5), if we denote for simplicity $f(z, v):=$ $K(z) \frac{|v|^{2^{*}-2} v}{d^{2 *} \beta}$ and $F(z, v):=\int_{0}^{v} f(z, t) \mathrm{d} t$, it holds

$$
\begin{aligned}
\int_{\Omega_{r_{n}}} f(z, v) Z v \mathrm{~d} z= & \int_{\Omega_{r_{n}}} Z(F(z, v)) \mathrm{d} z-\int_{\Omega_{r_{n}}}\left\langle Z, \nabla_{z} F(z, v)\right\rangle \mathrm{d} z \\
= & -\int_{\Omega_{r_{n}}} \operatorname{div} Z F(z, v) \mathrm{d} z+\int_{\partial \Omega_{r_{n}}} F(z, v)\langle Z, v\rangle \mathrm{d} \sigma \\
& -\int_{\Omega_{r_{n}}}\left\langle Z, \nabla_{z} F(z, v)\right\rangle \mathrm{d} z .
\end{aligned}
$$

Now, taking into account that $F(z, v)=\frac{1}{2^{*}} K(z) \frac{|v|^{2^{*}}}{d^{2^{*} \beta}}$ and that $\operatorname{div} Z=Q$, from (5.11), we get

$$
\begin{aligned}
\int_{\Omega_{r_{n}}} K(z) \frac{|v|^{2^{*}-2} v}{d^{2^{*} \beta}} Z v \mathrm{~d} z= & \left(\beta-\frac{Q}{2^{*}}\right) \int_{\Omega_{r_{n}}} K(z) \frac{|v|^{2^{*}}}{d^{2^{*} \beta}} \mathrm{d} z \\
& +\frac{1}{2^{*}} \int_{\partial \Omega_{r_{n}}} K(z) \frac{|v|^{2^{*}}}{d^{2^{*} \beta}}\langle Z, v\rangle \mathrm{d} \sigma-\frac{1}{2^{*}} \int_{\Omega_{r_{n}}} Z K \frac{|v|^{2^{*}}}{d^{2^{*} \beta}} \mathrm{d} z,
\end{aligned}
$$

where we have used that $\langle Z, \nabla d\rangle=d$, as observed in (2.5). So, inserting (5.6) and (5.12) into (5.5), we obtain

$$
\begin{aligned}
& \left(\frac{Q}{2^{*}}-\beta\right) \int_{\Omega_{r_{n}}} K(z) \frac{|v|^{2^{*}}}{d^{2^{*} \beta}} \mathrm{d} z+\frac{1}{2^{*}} \int_{\Omega_{r_{n}}} Z K \frac{|v|^{2^{*}}}{d^{2^{*} \beta}} \mathrm{d} z-\frac{1}{2^{*}} \int_{\partial \Omega_{r_{n}}} K(z) \frac{|v|^{2^{*}}}{d^{2 * \beta}}\langle Z, v\rangle \mathrm{d} \sigma \\
& =\frac{Q-2-2 \beta}{2} \int_{\Omega_{r_{n}}} d^{-2 \beta}\left|\nabla_{\alpha} v\right|^{2} \mathrm{~d} z-\frac{1}{2} \int_{\partial \Omega_{r_{n}}} d^{-2 \beta}\left|\nabla_{\alpha} v\right|^{2}\langle Z, v\rangle \mathrm{d} \sigma \\
& \quad+\int_{\partial \Omega_{r_{n}}} d^{-2 \beta}\left\langle\nabla_{\alpha} v, v_{\alpha}\right\rangle Z v \mathrm{~d} \sigma .
\end{aligned}
$$

On the other hand, multiplying Eq. (5.1) by $v$ and integrating by parts on $\Omega_{r_{n}}$, we have

$$
\int_{\Omega_{r_{n}}} K(z) \frac{|v|^{2^{*}}}{d^{2^{*} \beta}} \mathrm{d} z=\int_{\Omega_{r_{n}}} d^{-2 \beta}\left|\nabla_{\alpha} v\right|^{2} \mathrm{~d} z-\int_{\partial \Omega_{r_{n}}} d^{-2 \beta} v\left\langle\nabla_{\alpha} v, v_{\alpha}\right\rangle \mathrm{d} \sigma .
$$

So, by (5.13) and (5.14), and since $\frac{Q}{2^{*}}-\beta-\frac{Q-2-2 \beta}{2}=0$, we get

$$
\begin{aligned}
\frac{1}{2^{*}} & \int_{\Omega_{r_{n}}} Z K \frac{|v|^{2^{*}}}{d^{2^{*} \beta}} \mathrm{d} z-\frac{1}{2^{*}} \int_{\partial \Omega_{r_{n}}} K(z) \frac{|v|^{2^{*}}}{d^{2^{*} \beta}}\langle Z, v\rangle \mathrm{d} z \\
= & \frac{Q-2-2 \beta}{2} \int_{\partial \Omega_{r_{n}}} d^{-2 \beta} v\left\langle\nabla_{\alpha} v, v_{\alpha}\right\rangle \mathrm{d} \sigma-\frac{1}{2} \int_{\partial \Omega_{r_{n}}} d^{-2 \beta}\left|\nabla_{\alpha} v\right|^{2}\langle Z, v\rangle \mathrm{d} \sigma \\
& +\int_{\partial \Omega_{r_{n}}} d^{-2 \beta}\left\langle\nabla_{\alpha} v, v_{\alpha}\right\rangle Z v \mathrm{~d} \sigma .
\end{aligned}
$$

Now, let us pass to the limit as $r_{n} \rightarrow 0$ in (5.15). From the integrability of $Z K \frac{|v|^{2^{*}}}{d^{2^{*} \beta}}$, it follows that

$$
\frac{1}{2^{*}} \int_{\Omega_{r_{n}}} Z K \frac{|v|^{2^{*}}}{d^{2^{*} \beta}} \mathrm{d} z \longrightarrow \frac{1}{2^{*}} \int_{\Omega} Z K \frac{|v|^{2^{*}}}{d^{2^{*} \beta}} \mathrm{d} z, \quad \text { as } r_{n} \rightarrow 0 .
$$


Moreover, we verify that the boundary integrals on $\partial B_{r_{n}}$ in (5.15) vanish as $r_{n} \rightarrow 0$. Indeed, observe that, since $v=-\frac{\nabla d}{|\nabla d|}$ on $\partial B_{r_{n}}$, then

$$
\langle Z, v\rangle=-\frac{Z d}{|\nabla d|}=-\frac{d}{|\nabla d|} \text { on } \partial B_{r_{n}} .
$$

From this, and using (5.4), we have

$$
\begin{aligned}
& \int_{\partial B_{r_{n}}}\left(\frac{1}{2^{*}} K(z) \frac{|v|^{2^{*}}}{d^{2^{*} \beta}}-\frac{1}{2} d^{-2 \beta}\left|\nabla_{\alpha} u\right|^{2}\right)|\langle Z, v\rangle| \mathrm{d} \sigma \\
& \quad=r_{n} \int_{\partial B_{r_{n}}}\left(\frac{1}{2^{*}} K(z) \frac{|v|^{2^{*}}}{d^{2 * \beta}}-\frac{1}{2} d^{-2 \beta}\left|\nabla_{\alpha} u\right|^{2}\right) \frac{1}{|\nabla d|} \mathrm{d} \sigma \longrightarrow 0, \quad \text { as } r_{n} \rightarrow 0 .
\end{aligned}
$$

For the first integral in the right-hand side of (5.15), observe that

$$
\left|\left\langle\nabla_{\alpha} v, v_{\alpha}\right\rangle\right|=\left|\left\langle\nabla_{\alpha} v, \frac{\nabla_{\alpha} d}{|\nabla d|}\right\rangle\right| \leq \psi \frac{\left|\nabla_{\alpha} v\right|}{|\nabla d|} \quad \text { on } \partial B_{r_{n}} .
$$

Then, by (5.18), taking into account that $\psi \in L^{\infty}$ and using Hölder's inequality, we get

$$
\begin{aligned}
& \left|\int_{\partial B_{r_{n}}} d^{-2 \beta} v\left\langle\nabla_{\alpha} v, v_{\alpha}\right\rangle \mathrm{d} \sigma\right| \leq C \int_{\partial B_{r_{n}}} d^{-2 \beta} v \frac{\left|\nabla_{\alpha} v\right|^{2}}{|\nabla d|} \mathrm{d} \sigma \\
& \quad \leq C\left(\int_{\partial B_{r_{n}}} \frac{1}{|\nabla d|} \mathrm{d} \sigma\right)^{\frac{2^{*}-2}{2 \cdot 2^{*}}}\left(\int_{\partial B_{r_{n}}} \frac{|v|^{2^{*}}}{d^{2^{*} \beta}} \frac{1}{|\nabla d|} \mathrm{d} \sigma\right)^{\frac{1}{2^{*}}}\left(\int_{\partial B_{r_{n}}} \frac{\left|\nabla_{\alpha} v\right|^{2}}{d^{2 \beta}} \frac{1}{|\nabla d|} \mathrm{d} \sigma\right)^{\frac{1}{2}} \\
& =C\left(r_{n} \int_{\partial B_{r_{n}}} \frac{|v|^{2^{*}}}{d^{2 *} \beta} \frac{1}{|\nabla d|} \mathrm{d} \sigma\right)^{\frac{1}{2^{*}}}\left(r_{n} \int_{\partial B_{r_{n}}} \frac{\left|\nabla_{\alpha} v\right|^{2}}{d^{2 \beta}} \frac{1}{|\nabla d|} \mathrm{d} \sigma\right)^{\frac{1}{2}} \\
& =o(1), \quad \text { as } r_{n} \rightarrow 0,
\end{aligned}
$$

where we have used the fact that, by Federer's coarea formula [7], for $r>0$,

$$
\int_{\partial B_{r}} \frac{1}{|\nabla d|} \mathrm{d} \sigma=c_{Q^{r}}{ }^{Q-1}
$$

and we have concluded by means of (5.4).

Finally, concerning the integral $\int_{\partial B_{r_{n}}} d^{-2 \beta}\left\langle\nabla_{\alpha} v, v_{\alpha}\right\rangle Z v \mathrm{~d} \sigma$, note that the vector field $Z$ can be expressed as

$$
Z=\frac{d}{\psi^{2}} A_{\alpha} \nabla d
$$

as pointed out in [14, formula (2.13)] (see also [16, Proposition 3.1]). So, by (5.21), we get

$$
|Z v|=|\langle Z, \nabla v\rangle|=\left|\left\langle\frac{d}{\psi^{2}} \nabla_{\alpha} d, \nabla_{\alpha} v\right\rangle\right| \leq \frac{d}{\psi}\left|\nabla_{\alpha} v\right| \text { in } \Omega \backslash\{0\} .
$$

Hence, by (5.18) and (5.22), and using again (5.4), we obtain that

$$
\left|\int_{\partial B_{r_{n}}} d^{-2 \beta}\left\langle\nabla_{\alpha} v, v_{\alpha}\right\rangle Z v \mathrm{~d} \sigma\right| \leq r_{n} \int_{\partial B_{r_{n}}} d^{-2 \beta} \frac{\left|\nabla_{\alpha} v\right|^{2}}{|\nabla d|} \mathrm{d} \sigma \longrightarrow 0, \quad \text { as } r_{n} \rightarrow 0 .
$$

So, letting $r_{n} \rightarrow 0$ in (5.15) and using (5.16), (5.17), (5.19) and (5.23), the conclusion follows. 
As a consequence of the above Pohozaev-type identity, we prove the following nonexistence result on bounded starshaped domains of $\mathbb{R}^{N}$.

Theorem 5.2 Let $\Omega \subset \mathbb{R}^{N}$ be a connected bounded domain with $C^{1}$-boundary, $\delta_{\lambda}$ starshaped about the origin. Assume that $K \in C^{1}(\bar{\Omega})$ and that $Z K \leq 0$ in $\Omega$. Then, the problem

$$
-\operatorname{div}_{\alpha}\left(d^{-2 \beta} \nabla_{\alpha} v\right)=K(z) \frac{v^{2^{*}-1}}{d^{2^{*} \beta}} \quad \text { in } \Omega, \quad v=0 \text { on } \partial \Omega
$$

has no nonnegative nontrivial solutions $v \in \mathcal{D}_{\alpha}^{1,2}\left(\Omega, d^{-2 \beta} \mathrm{d} z\right) \cap \Lambda^{2}(\bar{\Omega} \backslash\{0\})$.

Proof Denoted by $\Pi:=\{x=0\}$ the degeneration set of the Grushin vector fields, we have that $v \in C^{1}(\bar{\Omega} \backslash \Pi)$, since $X_{j} v \in C^{1}(\bar{\Omega} \backslash\{0\})$ by assumption and the coefficients of the vector fields $X_{j}$ are $C^{1}$ and different from 0 in $\mathbb{R}^{N} \backslash \Pi$. Thus, the condition $v=0$ on $\partial \Omega$ implies

$$
\nabla v=\frac{\partial v}{\partial v} v \quad \text { on } \partial \Omega \backslash \Pi
$$

This gives that

$$
\begin{aligned}
\int_{\partial \Omega} d^{-2 \beta}\left\langle\nabla_{\alpha} v, v_{\alpha}\right\rangle Z v \mathrm{~d} \sigma & =\int_{\partial \Omega} d^{-2 \beta} \frac{\partial v}{\partial v}\left\langle\nabla_{\alpha} v, v_{\alpha}\right\rangle\langle Z, v\rangle \mathrm{d} \sigma \\
& =\int_{\partial \Omega} d^{-2 \beta}\left|\nabla_{\alpha} v\right|^{2}\langle Z, v\rangle \mathrm{d} \sigma .
\end{aligned}
$$

Hence, in this case, identity (5.2) reduces to

$$
\frac{1}{2^{*}} \int_{\Omega} Z K \frac{v^{2^{*}}}{d^{2^{*} \beta}} \mathrm{d} z=\frac{1}{2} \int_{\partial \Omega} d^{-2 \beta}\left|\nabla_{\alpha} v\right|^{2}\langle Z, v\rangle \mathrm{d} \sigma .
$$

So, from the assumption $Z K \leq 0$, we get

$$
\int_{\partial \Omega} d^{-2 \beta}\left(\frac{\partial v}{\partial v}\right)^{2}\left|v_{\alpha}\right|^{2}\langle Z, v\rangle \mathrm{d} \sigma \leq 0 .
$$

Then, since $\langle Z, v\rangle \geq 0$ on $\partial \Omega$ by the starshapedness of $\Omega$, we have

$$
\left(\frac{\partial v}{\partial v}\right)^{2}\langle Z, v\rangle=0 \text { at any point of } \partial \Omega .
$$

On the other hand, since $\Omega$ is bounded, there exists a bounded and connected open set $U \subset \mathbb{R}^{N} \backslash \Pi$ such that $\langle Z, v\rangle>0$ on $\partial \Omega \cap U$. Thus,

$$
\nabla v=\frac{\partial v}{\partial v} v \equiv 0 \quad \text { on } \partial \Omega \cap U .
$$

Setting $v \equiv 0$ in $\left(\mathbb{R}^{N} \backslash \Omega\right) \cap U$, we then obtain a nonnegative weak solution to

$$
-\operatorname{div}_{\alpha}\left(d^{-2 \beta} \nabla_{\alpha} v\right)=V v \text { in } U,
$$

where $V=K(z) \frac{v^{2^{*}-2}}{d^{2^{*} \beta}} \in L^{\infty}(U)$. So, by Proposition 2.5 in [20], $v \equiv 0$ in $U$. Finally, a connectedness argument gives $v \equiv 0$ in $\Omega$.

Proof of Theorem 1.4 The thesis follows from Theorem 5.2, taking into account the definition of the auxiliary function $v$ in (3.3). 
Finally, we prove a necessary condition for the existence of entire solutions to problem (5.1), involving the radial derivative $Z K$ of the coefficient $K$. The result extends to the Grushin context the Euclidean result in [10].

Theorem 5.3 Let $v \in \mathcal{D}_{\alpha}^{1,2}\left(\mathbb{R}^{N}, d^{-2 \beta} \mathrm{d} z\right)$ be a positive solution to

$$
-\operatorname{div}_{\alpha}\left(d^{-2 \beta} \nabla_{\alpha} v\right)=K(z) \frac{v^{2^{*}-1}}{d^{2^{*} \beta}} \text { in } \mathbb{R}^{N},
$$

where $K \in L^{\infty}\left(\mathbb{R}^{N}\right) \cap C^{1}\left(\mathbb{R}^{N}\right)$ and $Z K \in L^{\infty}\left(\mathbb{R}^{N}\right)$. Then,

$$
\int_{\mathbb{R}^{N}} Z K \frac{v^{2^{*}}}{d^{2 * \beta}} \mathrm{d} z=0
$$

Proof Let $v$ be a positive solution to (5.26). We shall apply identity (5.2) to $v$ on an appropriate sequence of balls $B_{R_{n}}$, with $R_{n} \rightarrow \infty$. To this aim, note that

$$
\int_{0}^{\infty} \mathrm{d} s \int_{\partial B_{s}}\left(K(z) \frac{v^{2^{*}}}{d^{2^{*} \beta}}+\frac{\left|\nabla_{\alpha} v\right|^{2}}{d^{2 \beta}}\right) \frac{1}{|\nabla d|} \mathrm{d} \sigma=\int_{\mathbb{R}^{N}}\left(K(z) \frac{v^{2^{*}}}{d^{2^{*} \beta}}+\frac{\left|\nabla_{\alpha} v\right|^{2}}{d^{2 \beta}}\right) \mathrm{d} z<\infty .
$$

Therefore, there exists a sequence $R_{n} \rightarrow+\infty$ such that

$$
R_{n} \int_{\partial B_{R_{n}}}\left(K(z) \frac{v^{2^{*}}}{d^{2 *} \beta}+\frac{\left|\nabla_{\alpha} v\right|^{2}}{d^{2 \beta}}\right) \frac{1}{|\nabla d|} \mathrm{d} \sigma \longrightarrow 0, \quad \text { as } n \rightarrow \infty .
$$

Now, reasoning as in the previous proof, identity (5.2) on the balls $B_{R_{n}}$ takes the form

$$
\begin{aligned}
\frac{1}{2^{*}} & \int_{B_{R_{n}}} Z K \frac{v^{2^{*}}}{d^{2^{*} \beta}} \mathrm{d} z-\frac{R_{n}}{2^{*}} \int_{\partial B_{R_{n}}} K(z) \frac{v^{2^{*}}}{d^{2 *} \beta} \frac{1}{|\nabla d|} \mathrm{d} \sigma \\
= & \frac{Q-2-2 \beta}{2} \int_{\partial B_{R_{n}}} d^{-2 \beta} v\left\langle\nabla_{\alpha} v, \frac{\nabla_{\alpha} d}{|\nabla d|}\right\rangle \mathrm{d} \sigma \\
& -\frac{R_{n}}{2} \int_{\partial B_{R_{n}}} d^{-2 \beta}\left|\nabla_{\alpha} v\right|^{2} \frac{1}{|\nabla d|} \mathrm{d} \sigma+R_{n} \int_{\partial B_{R_{n}}} d^{-2 \beta} \frac{1}{\psi^{2}}\left(\left\langle\nabla_{\alpha} v, \nabla_{\alpha} d\right\rangle\right)^{2} \frac{1}{|\nabla d|} \mathrm{d} \sigma .
\end{aligned}
$$

Hence, estimating the boundary integrals in (5.30) as in the previous proof and passing to the limit as $R_{n} \rightarrow \infty$, the thesis follows by means of (5.29).

Proof of Theorem 1.5 The result follows from Theorem 5.3, taking into account the definition of $v$ in (3.3).

Acknowledgements The author is a member of Gruppo Nazionale per l'Analisi Matematica, la Probabilità e le loro Applicazioni (GNAMPA) of the Istituto Nazionale di Alta Matematica (INdAM), and she is partially supported by the GNAMPA research Project 2018 "Metodi di analisi armonica e teoria spettrale per le equazioni dispersive."

\section{References}

1. Beckner, W.: On the Grushin operator and hyperbolic symmetry. Proc. Am. Math. Soc. 129(4), 1233-1246 (2001)

2. Bieske, T., Gong, J.: The p-Laplace equation on a class of Grushin-type spaces. Proc. Am. Math. Soc. 134(12), 3585-3594 (2006) 
3. Cao, D., Han, P.: Solutions to critical elliptic equations with multi-singular inverse square potentials. J. Differ. Equ. 224, 332-372 (2006)

4. D’Ambrosio, L.: Hardy inequalities related to Grushin-type operators. Proc. Am. Math. Soc. 132(3), 725-734 (2004)

5. D’Ambrosio, L., Lucente, S.: Nonlinear Liouville theorems for Grushin and Tricomi operators. J. Differ. Equ. 193(2), 511-541 (2003)

6. Dou, J., Niu, P.: Hardy-Sobolev type inequalities for generalized Baouendi-Grushin operators. Miskolc Math. Notes 8(1), 73-77 (2007)

7. Federer, H.: Geometric Measure Theory, Die Grundlehren der mathematischen Wissenschaften, vol. 153. Springer, New York (1969)

8. Felli, V., Ferrero, A., Terracini, S.: Asymptotic behavior of solutions to Schrödinger equations near an isolated singularity of the electromagnetic potential. J. Eur. Math. Soc. 13, 119-174 (2011)

9. Felli, V., Schneider, M.: A note on regularity of solutions to degenerate elliptic equations of CaffarelliKohn-Nirenberg type. Adv. Nonlinear Stud. 3(4), 431-443 (2003)

10. Felli, V., Schneider, M.: Compactness and existence results for degenerate critical elliptic equations. Commun. Contemp. Math. 7, 37-73 (2005)

11. Franchi, B., Lanconelli, E.: Une metrique associée à une classe d'opérateurs elliptiques dégénérés. In: Proceedings of the Meeting "Linear Partial and Pseudodifferential Operators", Rend. Sem. Mat. Univ. e Politec. Torino (1982), pp. 105-114 (1982)

12. Franchi, B., Lanconelli, E.: Hölder regularity theorem for a class of linear nonuniformly elliptic operators with measurable coefficients. Ann. Sc. Norm. Sup Pisa Cl. Sci. 10(4), 523-541 (1983)

13. Franchi, B., Lanconelli, E.: An embedding theorem for Sobolev spaces related to non-smooth vector fields and Harnack inequality. Commun. Partial Differ. Equ. 9, 1237-1264 (1984)

14. Garofalo, N.: Unique continuation for a class of elliptic operators which degenerate on a manifold of arbitrary codimension. J. Differ. Equ. 104, 117-146 (1993)

15. Garofalo, N., Lanconelli, E.: Existence and nonexistence results for semilinear equations on the Heisenberg group. Indiana Univ. Math. J. 41, 71-98 (1992)

16. Garofalo, N., Vassilev, D.: Strong unique continuation properties of generalized Baouendi-Grushin operators. Comm. Partial Differ. Equ. 32(4-6), 643-663 (2007)

17. Han, P.: Asymptotic behavior of solutions to semilinear elliptic equations with Hardy potential. Proc. Am. Math Soc. 135, 365-372 (2007)

18. Jannelli, E.: The role played by space dimension in elliptic critical problems. J. Differ. Equ. 156, 407-426 (1999)

19. Kogoj, A.E., Lanconelli, E.: X-elliptic operators and X-control distances. Contributions in honor of the memory of Ennio De Giorgi. Ric. Mat. 49(Suppl.), 223-243 (2000)

20. Kogoj, A.E., Lanconelli, E.: On semilinear $\Delta_{\lambda}$-Laplace equation. Nonlinear Anal. 75, 4637-4649 (2012)

21. Kogoj, A.E., Sonner, S.: Hardy type inequalities for $\Delta_{\lambda}$-Laplacians. Complex Var. Elliptic Equ. 61(3), 422-442 (2016)

22. Kombe, I.: On the nonexistence of positive solutions to doubly nonlinear equations for Baouendi-Grushin operators. Discrete Contin. Dyn. Syst. 33(11-12), 5167-5176 (2013)

23. Lascialfari, F., Pardo, D.: Compact embedding of a degenerate Sobolev space and existence of entire solutions to a semilinear equation for a Grushin-type operator. Rend. Sem. Mat. Univ. Padova 107, 139152 (2002)

24. Loiudice, A.: Sobolev inequalities with remainder terms for sublaplacians and other subelliptic operators. NoDEA Nonlinear Differ. Equ. Appl. 13, 119-136 (2006)

25. Loiudice, A.: Asymptotic behaviour of solutions for a class of degenerate elliptic critical problems. Nonlinear Anal. 70(8), 2986-2991 (2009)

26. Loiudice, A.: $L^{p}$-weak regularity and asymptotic behavior of solutions for critical equations with singular potentials on Carnot groups. NoDEA Nonlinear Differ. Equ. Appl. 17, 575-589 (2010)

27. Loiudice, A.: Critical growth problems with singular nonlinearities on Carnot groups. Nonlinear Anal. 126, 415-436 (2015)

28. Loiudice, A.: Local behavior of solutions to subelliptic problems with Hardy potential on Carnot groups. Mediterr. J. Math. 15(3), 20 (2018) (Art. 81)

29. Loiudice, A.: Optimal decay of $p$-Sobolev extremals on Carnot groups. J. Math. Anal. Appl. 470(1), 619-631 (2019)

30. Luyen, D.T., Tri, N.M.: Existence of infinitely many solutions for semilinear degenerate Schrödinger equations. J. Math. Anal. Appl. 461(2), 1271-1286 (2018)

31. Mokrani, H.: Semilinear subelliptic equations on the Heisenberg group with a singular potential. Commun. Pure Appl. Math. 8, 1619-1636 (2009) 
32. Monti, R.: Sobolev inequalities for weighted gradients. Commun. Partial Differ. Equ. 31, 1479-1504 (2006)

33. Monti, R., Morbidelli, D.: Kelvin transform for Grushin operators and critical semilinear equations. Duke Math. J. 131, 167-202 (2006)

34. Monticelli, D.: Maximum principles and the method of moving planes for a class of degenerate elliptic linear operators. J. Eur. Math. Soc. 12, 611-654 (2010)

35. Pohozaev, S.I.: Eigenfunctions of the equation $\Delta u+\lambda f(u)=0$. Dokl. Akad. Nauk. SSSR 165(1), 33-36 (1965)

36. Smets, D.: Nonlinear Schrödinger equations with Hardy potential and critical nonlinearities. Trans. Am. Math. Soc. 375, 2909-2938 (2005)

37. Terracini, S.: On positive entire solutions to a class of equations with singular coefficient and critical exponent. Adv. Differ. Equ. 1, 241-264 (1996)

38. Thuy, P.T., Tri, N.M.: Nontrivial solutions to boundary value problems for semilinear strongly degenerate elliptic differential equations. NoDEA Nonlinear Differ. Equ. Appl. 19, 279-298 (2012)

39. Tri, N.M.: On the Grushin equation. Mat. Zamet. 63(1), 95-105 (1998)

40. Wang, C., Wang, Q., Yang, J.: On the Grushin critical problem with a cylindrical symmetry. Adv. Differ. Equ. 20(1-2), 77-116 (2015)

41. Yang, Q., Su, D., Kong, Y.: Improved Hardy inequalities for Grushin operators. J. Math. Anal. Appl. 424, 321-343 (2015)

Publisher's Note Springer Nature remains neutral with regard to jurisdictional claims in published maps and institutional affiliations. 